\title{
Prise de mesures pour les adolescentes en periode de la COVID-19: implications pour la programmation des espaces sûrs au Sahel
}

Population Council

Follow this and additional works at: https://knowledgecommons.popcouncil.org/departments_sbsr-pgy How does access to this work benefit you? Let us know!

\section{Recommended Citation}

"Prise de mesures pour les adolescentes en periode de la COVID-19: implications pour la programmation des espaces sûrs au Sahel," SWEDD Project Brief. New York: Population Council, 2021. 


\section{PRISE DE MESURES POUR LES ADOLESCENTES EN PERIODE DE LA COVID-19; IMPLICATIONS POUR LA PROGRAMMATION DES ESPACES SÛRS AU SAHEL}

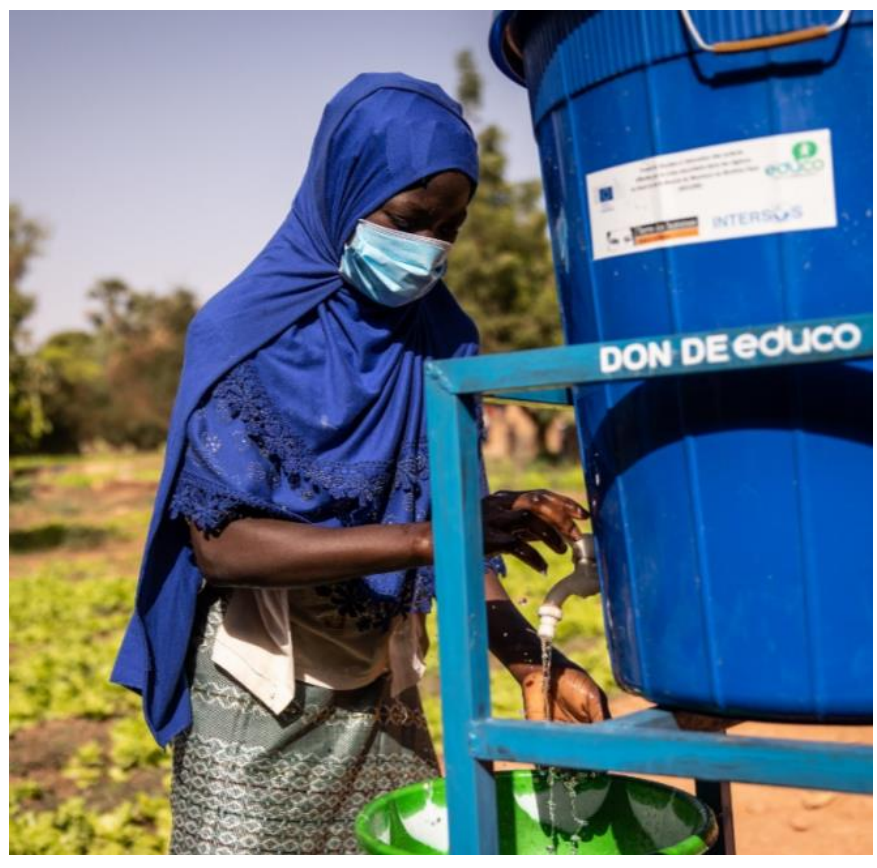

Crédit photo: Olympia de Maismont, European Union. Licensed under $\underline{\text { CC BY-NC-ND } 4.0}$

\section{RECOMMANDATIONS}

1. Modifier les configurations actuelles des espaces sûrs qui se tiennent en personne

2. Contacter les adolescentes par téléphone

3. Mettre l'accent sur la capacité des mentores à diffuser des messages de bonnes pratiques et à partager des produits de santé

4. Élargir les priorités du programme afin de faire face à d'éventuels chocs

5. Renforcement du potentiel économique

Ce document présente les stratégies utilisées par les organisations ayant mis en œuvre des programmes axés sur les adolescents et jeunes filles (AJF) en période de crises. Il vise à informer les organisateurs du programme SWEDD ainsi que leurs partenaires de mise en œuvre sur comment maintenir et/ou adapter la bonne programmation des espaces sûrs pendant la

pandémie de la COVID-19 tout en gardant le contact avec les AJF et en continuant à leur apporter le soutien dont elles ont besoin. SWEDD se doit d'être un moteur de changement en accordant une priorité particulière à la sécurité des AJF et des mentores dans le contexte de la pandémie de la COVID-19 et pendant d'autres crises.

Les stratégies ci-dessous ont été utilisées, et dans certains cas étudiés de manière approfondie, pour adapter les programmes des AJF tels que les programmes actuels des espaces sûrs aux différentes contraintes imposées par la COVID-19. Sur la base de ces expériences, nous incluons des recommandations de stratégies prometteuses qui permettent d'" apprendre en faisant " la mise en œuvre des projets comme le SWEDD en période de crises. Les unités de gestion du SWEDD, les partenaires de mise en œuvre, les représentants des ministères, et les partenaires d'assistance technique devraient prendre ces recommandations en compte et savoir les appliquer à leurs différents contextes. Toutes ces recommandations devraient pouvoir être adaptées à chaque pays et à chacune des populations cibles. 


\section{LES EFFETS DE LA COVID-19 SUR LES ADOLESCENTES ET JEUNES FILLES}

La pandémie mondiale de la COVID-19 a eu des effets de risques primaires (c'est-à-dire l'infection par la COVID) et de risques secondaires (comme la fermeture des écoles, le confinement et l'instabilité économique des ménages) sur les communautés, les familles et sur les AJF en particulier.

Dans la région du Sahel, les gouvernements et les ONG des pays comme le Burkina Faso, le Mali et le Niger ont signalé une augmentation du besoin d'aide humanitaire pour les enfants. ${ }^{1}$

Les difficultés de longue date qui pèsent sur le bienêtre des filles, et l'inégalité entre les sexes signifient que ces circonstances sont susceptibles d'avoir un impact disproportionné sur les AJF. ${ }^{2}$ Une enquête de CARE sur les effets de la COVID-19 sur les normes du genre dans 12 pays d'Afrique de l'Ouest a révélé un accès réduit aux soins de santé ( $52 \%$ des femmes, $42 \%$ des hommes), y compris aux services de santé sexuelle et reproductive, en raison de la méfiance envers les agents de santé et de la peur de contracter la COVID-19. ${ }^{3}$ Les études menées ailleurs sur les adolescents révèlent de faibles niveaux de connaissance de la transmission asymptomatique de la COVID-19 ainsi que le respect des consignes de protection (par exemple le port de masques) et les menaces de la pandémie sur l'éducation, la sécurité alimentaire et la santé mentale des adolescents.4,5 || serait également approprié de mener des recherches sur les différences entre les sexes en ce qui concerne les effets de la COVID-19 dans les communautés pastorales de la région du Sahel. Dans ces communautés, les femmes et les filles restent à la maison tandis que les hommes et les garçons se déplacent avec le bétail. Le risque d'exposition au COVID-19 et les effets secondaires sont susceptibles de différer pour les filles et les garçons en raison des différences qui existent en termes d'accès à la terre pour le pâturage. $6,7,8$
COMMENT ASSURER UNE CONTINUITE DES PROGRAMMES DES ESPACES SÛRS PENDANT LA COVID-19

Les programmes axés sur les AJF dans les pays à revenus faibles et intermédiaires ont utilisé différentes stratégies pour atténuer les risques primaires et secondaires de la COVID-19, notamment en réajustant leurs structures et/ou leurs objectifs pour faire face à la situation. Les exemples suivants illustrent les types de tactiques utilisées par les organisations dans des contextes présentant des caractéristiques similaires à la région du Sahel. Ils peuvent fournir des leçons utiles pour la programmation du SWEDD. II est essentiel d'adapter ces approches aux caractéristiques spécifiques du contexte du programme et de la sous-population qui est ciblée ou du segment des AJF dont il est question. Pour plus d'informations sur la segmentation hétérogène des AJF pour les programmes adaptés, veuillez-vous référer à Le Guide des Normes Minimales pour des Espaces Sûrs. Les initiateurs doivent adapter leur planification en fonctions des besoins les plus urgents des adolescentes afin de s'assurer que les programmes SWEDD peuvent continuer à soutenir et à protéger les AJF les plus vulnérables pendant les crises comme celle de la COVID-19.

\section{MODIFIER LES CONFIGURATIONS ACTUELLES DES ESPACES SURS}

\section{Recommandations}

- Opter pour des espaces sûrs qui se tiennent à l'extérieur tout en continuant à s'assurer du respect de la vie privée des mentores et des participants.

- Organiser des réunions de groupe plus restreintes, où les mesures barrières sont respectées et où le port du masque est obligatoire

- Organiser des sessions hebdomadaires supplémentaires et multiples en petits groupes pour permettre au nombre initial des participantes d'avoir accès au programme

\footnotetext{
${ }^{1}$ UN News. Violence, COVID-19, Contribute to Rising Humanitarian Needs in the Sahel. https://news.un.org/en/story/2020/10/10756.

${ }^{2}$ UN News. Violence, COVID-19, Contribute to Rising Humanitarian Needs in the Sahel. https://news.un.org/en/story/2020/10/1075602.

${ }^{3}$ Relief Web. (2021). New CARE report: how COVID-19 is changing gender norms in West Africa. https://reliefweb.int/report/benin/new-care-reporthow-covid-19-changing-gender-norms-west-africa.

4 "Social, health, education and economic effects of COVID -19 on adolescent girls in Kenya: Responses from the first round of adolescent data collection, August 2020-Wajir," COVID-19 Research \& Evaluations brief. Nairobi: Population Council, 2020
}

\footnotetext{
${ }^{5}$ Amin, Sajeda, Ubaidur Rob, Sigma Ainul, Md. Irfan Hossain, Forhana Rahman Noor, Iqbal Ehsan, and Mehnaz Manzur. 2020. "Bangladesh: COVID-19 Knowledge, Attitudes, Practices \& Needs-Responses from three rounds of data collection among adolescent girls in districts with high rates of child marriage," COVID-19 Research \& Evaluations brief. Dhaka: Population Council.

${ }^{8}$ USAID. (2021). USAID/Sahel Regional Office COVID-Specific Gender Analysis. https://reliefweb.int/report/benin/new-care-report-how-covid-19-changinggender-norms-west-africa.
} 


\section{Lecons tirées de l'expérience}

L'expérience des groupes à capacité réduite montre qu'il est possible de respecter les mesures barrières de santé tout en offrant aux AJF la possibilité de tisser des liens forts en personne. ${ }^{9}$ Après un arrêt initial de leurs activités, REPACTED au Kenya a repris les rencontres de groupes qu'ils ont limité à un maximum de 10 participants à la fois. Le modèle d'espace sûr modifié utilisant des groupes plus petits a également permis au projet Education as a Vaccine (EVA) du Nigéria de renouer avec les mentores et de réinitialiser les espaces sûrs pour les filles avec des groupes plus petits où les mesures barrières étaient respectées. ${ }^{10}$

Une autre stratégie pour protéger les mentores et les participants consiste à modifier le lieu et la structure des réunions afin de respecter les règles de santé locales et mondiales. La Fondation Batonga au Bénin a testé des espaces sûrs modifiés qui se réunissent à l'extérieur. Les participants suivent les recommandations nationales de distanciation sociale, les mentores portent des masques, et les activités et jeux ont été revus pour réduire au maximum le contact physique. ${ }^{11}$ Des informations supplémentaires sont partagées entre les participants et les mentores à l'aide d'enregistrements vocaux. Ces espaces sûrs modifiés ont été mis en place dans les zones rurales pendant une période où les cas de transmission communautaire était limitée à la capitale économique de Cotonou.

\section{Le Guide des Normes Minimales pour des Espaces}

$\underline{\text { Surrs }}^{12}$ souligne l'importance de reconnaître I'hétérogénéité des adolescentes en utilisant la " segmentation " pour subdiviser les groupes d'AJF en fonction de caractéristiques similaires. Un modèle adapté, avec des groupes de taille réduite, pourrait permettre d'appliquer la segmentation en organisant des sessions dédiées aux AJF qui partagent plusieurs caractéristiques (par exemple, les filles mariées, les filles non scolarisées âgées de 10 à 14 ans). Cette stratégie peut permettre d'adapter le contenu aux circonstances de vie distinctes des AJF ayant des expériences similaires.
COMMUNIQUER AVEC LES ADOLESCENTES PAR TELEPHONE

\section{$\underline{\text { Recommandations }}$}

- L'utilisation des smartphones peut être efficace pour maintenir le contact avec les AJF et veiller à l'apparition de risques secondaires quelconques ou d'autres besoins qui apparaissent au sein du contexte familial pendant la pandémie.

- Bien que certaines AJF aient accès à des téléphones portables en les empruntant à des amis ou aux membres de leur famille, elles sont moins susceptibles d'en posséder ou d'avoir accès par rapport aux jeunes garçons et aux adultes, ${ }^{13}$ en particulier les filles marginalisées comme les enfants domestiques.

- Tenez compte des facteurs contextuels qui facilitent ou pas l'accès aux téléphones portables par les populations et les normes sociales ou culturelles relatives à l'utilisation du téléphone portable par les AJF ; ces informations vous permettront d'être fixé sur la faisabilité d'atteindre les AJF les plus vulnérables par ce moyen.

- Utiliser les téléphones mobiles pour créer des possibilités d'engagement flexibles (par exemple des " défis " interactifs que les AJF doivent relever chaque semaine) afin d'accroître et/ou de maintenir la possibilité qu'offre le programme à entrer en contact avec les AJF.

- La souplesse des horaires et la multiplicité des canaux de communication (sms, appels téléphoniques et réunions en personne, etc.) sont des moyens susceptibles d'améliorer le succès du programme à travers l'utilisation des téléphones portables.

\section{Leçons tirées de l'expérience}

Les organisations mettant en œuvre les programmes destinés aux AJF sont en train de tester les meilleurs moyens de communiquer par téléphone avec les participantes pour faire passer les informations et les aider à mieux comprendre le programme. Au Kenya, une enquête sur les influences prépandémiques des AJF sur les transitions vers l'âge adulte pendant la pandémie de la COVID-19 a révélé qu'environ un tiers (32 \%) des AJF possédaient un téléphone mobile et qu'un plus grand pourcentage de filles plus âgées (15-19 ans) utilisaient des téléphones mobiles pour accéder aux travaux scolaires, par rapport aux plus

information on different models and experiences of girls' programs during COVID-19.

12 Population Council. 2021. Le Guide des normes minimales pour des espaces sûrs : la conception, la mise en œuvre, et le suivi, l'évaluation et l'apprentissage. New York.

https://www.popcouncil.org/uploads/pdfs/2021PGY GuideNormesMinimal esEspacesSurs.pdf

13 Girl Effect \& Vodafone Foundation. (2018). Real girls, real lives, connected. https://prdgirleffectcorp.s3.amazonaws.com/documents/GE_VO_Full_Reportcompressed.pdf

\footnotetext{
Prevention Guidance on Community Physical Distancing During COVID-19 Pandemic; Physical Distancing, Social Support"

during-covid-19-outbreak/

10 Briggs, Hannah, Nicole Haberland, Sapna Desai, Thomas de Hoop, and

Thoai Ngo. 2020. "The impact of COVID-19 on opportunities for adolescent girls and the role of girls' groups," brief, Evidence Consortium on Women's Groups.

${ }^{11}$ Adaptations in Crisis is a webinar presented by the Population Council's Adolescent Girls Community of Practice, focused on disseminating
} 
jeunes (10-14 ans) (34\% contre 24\%). ${ }^{14}$ Ces résultats confirment le potentiel de l'utilisation des téléphones portables pour atteindre les filles, en particulier les AJF plus âgées, bien que la plupart des personnes interrogées soient dans des familles où le téléphone portable est partagé entre tous.

Dans les cas de programmes où l'utilisation des téléphones mobiles est répandue, les responsables de la mise en œuvre ont organisé des sessions dirigées par les mentores par le biais d'appels téléphoniques de groupe. Dans le cadre du projet Keeping Girls in Schools to Reduce Child Marriage in Rural Bangladesh, les sessions comprenaient trois filles pendant 15 minutes deux fois par semaine. Au cours de ces sessions, les filles ont reçu des informations sur la santé sexuelle et reproductive, sur la COVID-19, sur l'éducation financière, mais aussi des cours d'anglais. Les défis initiaux liés aux horaires et à l'utilisation du téléphone ont été surmontés en permettant des choix d'horaires flexibles. 15

La Girl MOVE Academy au Mozambique a maintenu le contact avec les AJF par le biais d'appels téléphoniques et de SMS. Lors d'une période de confinement au Mozambique, le passage à l'utilisation des moyens de communication en ligne a entravé les efforts des mentores de rester en contact avec toutes les participantes du programme, car les filles n'avaient pas toutes accès à une connexion Internet fiable. ${ }^{16}$ Seuls $6 \%$ des participantes possédaient un téléphone portable, mais le personnel a réussi à se connecter avec près de $70 \%$ des filles au départ, ce chiffre passant à $80 \%$ avec le temps. Cette augmentation a été largement attribuée à la modification des tactiques de contact avec les AJF. Les mentores ont d'abord contacté les participantes par sms en leur posant des questions sur leurs connaissances en matière de santé, puis ont fait un suivi téléphonique quelques jours plus tard pour discuter du bien-être des filles et parler des questions reçues via sms. Au cours des trois premiers mois de la pandémie, ces appels ont été jumelés à des visites hebdomadaires respectant les normes de distanciation sociale. La fréquence des contacts avec les filles, ainsi que les diverses stratégies de sensibilisation (SMS, appels téléphoniques et visites en personne), ont contribué à renforcer les relations entre les filles et les mentores.
METTRE L'ACCENT SUR LA CAPACITE DES MENTORES A DIFFUSER DES MESSAGES DE BONNES PRATIQUES ET A PARTAGER DES PRODUITS DE SANTE

\section{Recommandations}

- Les mentores connues par les familles des AJF peuvent effectuer des visites à domicile pour maintenir le lien avec les participantes et les soutenir au besoin.

- Former les mentores aux mesures de prévention de la COVID-19, ainsi qu'aux messages sanitaires ou aux informations éducatives supplémentaires qu'elles devront livrer lors des visites des ménages.

- Les visites doivent respecter les règles locales visant à réduire la propagation de la COVID-19 (porter un masque, respecter la distanciation sociale, se rencontrer à l'extérieur dans un lieu privé si possible).

\section{Leçons tirées de l'expérience}

Les mentores des groupes communautaires, qui sont le plus souvent connues et respectés de tous localement, sont dans une position unique qui leur permettent de maintenir le contact avec les AJF surtout les plus vulnérables, pendant les périodes où les mouvements et les interactions au sein de la communauté sont limités. Tirer profit des capacités déjà existantes de ces mentores est une stratégie clé que les programmes axés sur les AJF devraient utiliser pour s'assurer du maintien des liens entre les mentores et les participantes, et de leur sécurité.

Dans les situations où les réunions de groupe ont cessé en raison des restrictions liées à la pandémie, les visites à domicile des mentores ont constitué une alternative pour maintenir le contact avec les AJF. La Fondation Batonga, au Bénin, a fait appel à ses mentores pour effectuer des visites à domicile auprès des participantes et leur fournir du savon pour se laver les mains et leur passer des informations sur la santé et I'hygiène. En Tanzanie, le programme communautaire Secondary Education for Girls' Advancement (SEGA) a également mobilisé des mentores pour fournir des connaissances sur les aptitudes de la vie quotidienne et distribuer des équipements de protection sanitaires individuelle comme des masques et du savon aux participantes du programme. ${ }^{17}$ Les mentores du programme

\footnotetext{
adolescent girls and the role of girls' groups," brief, Evidence Consortium on Women's Groups.

16 https://buildcommunity4girls.org/fieldexperience-covidresponse-2-mozambique/

17 Landskroner-Eiger, S. (2020). Nurturing Minds/SEGA SchoolDriven Response to Covid-19 in Tanzania. In

"And still they persist." - 8-Part Series on Community Protection and Revitalization from the Front

\footnotetext{
14 Bellerose, M., Diaw, M., Pinchoff, J., Kangwana, B., \& Austrian, K. (2020). CoVID-19, Girlhood Studies, 13(3), 133-150. Retrieved Apr 27, 2021, from https://www.berghahnjournals.

15 This project is a scale up of Bangladesh's BALIKA project. Briggs, Hannah, Nicole Haberland, Sapna Desai, Thomas de Hoop, and Thoai Ngo. 2020. "The impact of COVID-19 on opportunities for
} 
Secure Futures à Nairobi au Kenya, ont effectué des visites à domicile auprès d'adolescentes enceintes et de jeunes mères afin de vérifier leur bien-être et de les orienter vers des services adéquats pour un soutien psychosocial. ${ }^{18}$

\section{ÉLARGIR LES PRIORITES DU PROGRAMME AFIN DE FAIRE FACE A D'EVENTUELS CHOCS}

\section{Recommandations}

- Examinez comment les responsables de la mise en œuvre peuvent soutenir les AJF et leurs familles au-delà des objectifs initiaux du programme afin d'atténuer l'impact des chocs imprévus.

- Maintenir la priorité sur l'éducation des filles.

\section{Leçons tirées de l'expérience}

Les organisations œuvrant dans le cadre de programmes de soutien aux AJF ont envisagé des moyens de réagir aux chocs liés à la pandémie en ce qui concerne l'accès à la nourriture, l'emploi et la santé en général. Cela peut nécessiter qu'on élargisse la portée initiale du soutien que les AJF et leurs familles reçoivent. Les organisations ont envisagé certaines solutions pour continuer d'apporter leur soutien aux AJF et leurs familles audelà des buts initiaux de ces programmes centrés sur les filles afin de faire face aux problèmes qui surgissent. En Tanzanie par exemple, le SEGA a inclus une aide alimentaire aux familles dans les communautés où il travaille. ${ }^{19}$

Un des effets secondaires de la pandémie est que les filles sont obligées de rester à la maison pendant la fermeture des écoles, ce qui résulte à plus de responsabilités domestiques qui leur sont confiées. Plus la pandémie se prolonge, plus l'impact est important sur l'éducation des filles et leurs perspectives de retour à l'école. L'Académie Girl MOVE du Mozambique visait à prévenir ce problème d'abandon scolaire en connectant les filles à des programmes scolaires grâce au développement et à l'introduction d'un "jeu d'alphabétisation". Ce jeu

Lines of Covid-19 Response" by S. Soares. Adolescent Girls Community of Practice.

https://buildcommunity4girls.org/fieldexperience-covid-response5-tanzania/

18 Briggs, Hannah, Nicole Haberland, Sapna Desai, Thomas de Hoop, and Thoai Ngo. 2020. "The impact of COVID-19 on opportunities for adolescent girls and the role of girls' groups," brief, Evidence Consortium on Women's Groups.

19 Landskroner-Eiger, S. (2020). Nurturing Minds/SEGA SchoolDriven Response to Covid-19 in Tanzania. In "“And still they persist." - 8-Part Series on Community Protection and Revitalization from the Front Lines of Covid-19 Response" by S. Soares. Adolescent Girls Community of Practice.

https://buildcommunity4girls.org/fieldexperience-covid-response5-tanzania/ proposait des moyens de promouvoir l'intérêt des AJF pour l'éducation.

\section{RENFORCEMENT DU POTENTIEL ECONOMIQUE}

\section{Recommandations}

- En consultation avec les communautés et les AJF participant aux groupes d'espaces sûrs, le SWEDD peut identifier des moyens de soutenir les familles et les AJF qui font face à une instabilité économique et financière en raison de la pandémie.

\section{Leçons tirées de l'expérience}

II ne fait aucun doute que la pandémie a porté atteinte aux moyens de subsistance des AJF et de leurs familles. ${ }^{20}$ La mobilité réduite au sein des communautés et avec les autres, ainsi que les fermetures des marchés et les restrictions sur les rassemblements ont réduit les possibilités de générer des revenus. ${ }^{21}$ Une des conséquences possibles de ce changement de situation est que certaines familles se sont vues obligées de se séparer de leurs employés de maison. Ces travailleurs domestiques sont peut- être des AJF vulnérables susceptibles de se retrouver au chômage et sans abri, avec des options limitées pour leur bienêtre et leur survie, ce qui augmenterait les risques auxquels ce groupe déjà marginalisé est confronté. ${ }^{22}$

Les organisations réalisant des programmes pour les AJF travaillent déjà à élargir les opportunités économiques offertes aux AJF. Par exemple, le Centre des Arts en Haïti travaille avec des filles vulnérables pour fabriquer des masques qui seront distribués et vendus dans les communautés. Ils fournissent également une petite paie aux AJF pour entretenir les points de lavage de mains (se rassurer de l'approvisionnement en eau, fournir du savon, etc.) en plus de faire passer des messages pour promouvoir le lavage des mains dans la communauté. ${ }^{23}$ II est impératif qu'un suivi soit fait sur les activités génératrices de revenus pour les AJF afin d'évaluer comment les bénéfices (s'il y en a) sont utilisés, ou qui les reçoit.

\footnotetext{
20 Landskroner-Eiger, S. (2020.

21 CARE. (2020). COVID-19 could condemn women to decades of poverty: Implications of the COVID-19 pandemic on women's and girls' economic justice and rights. ReliefWeb. https://reliefweb.int/sites/reliefweb.int/files/resources/CARE Imp lications of COVID19 on WEE 300420.pdf

22 Briggs, Hannah, Nicole Haberland, Sapna Desai, Thomas de Hoop, and Thoai Ngo. 2020. "The impact of COVID-19 on opportunities for adolescent girls and the role of girls' groups," brief, Evidence Consortium on Women's Groups.

${ }^{23}$ Adaptations in Crisis is a webinar presented by the Population Council's Adolescent Girls Community of Practice, focused on disseminating information on different models and experiences of girls' programs during COVID-19.
} 